\title{
Well Differentiated
}

National Cancer Institute

\section{Source}

National Cancer Institute. Well Differentiated. NCI Thesaurus. Code C14171.

Describes tumor cells that generally retain the appearance of normal cells and tend to grow and spread at a slower rate than undifferentiated or poorly differentiated tumor cells. 